\title{
Enquêter sur la sexualité des néo-pentecotistes de la région parisienne
}

\author{
Retour sur l'expérience d'une socio-anthropologue \\ parmi les "siens"
}

Pamela Millet-Mouity ${ }^{1}$

\begin{abstract}
[Résumé] L'objectif de cet article est de discuter des enjeux méthodologiques et épistémologiques d'une recherche menée parmi les converti·es·s néo-pentecôtistes de la région parisienne. L'étude revient sur les conditions d'enquêtes menées au sein d'églises néo-pentecôtistes fréquentées majoritairement par des migrants africanocaribéens et leurs descendants, mettant en évidence l'ambivalence et les jeux de positionnements du chercheur. En cherchant à signaler en quoi les implications de l'ethnographe sur le terrain des religions, mais surtout en quoi sa proximité ethnique, culturelle, religieuse, voire sociale avec le milieu enquêté sont heuristiques pour les études sur le fait religieux, l'auteure montre comment la méthode basée sur le dévoilement de soi et la technique du cas par cas permettent de repenser plus largement la notion classique de «bonne distance » au cœur de la relation ethnographique.

Mots-clés : enquêtes de terrain, religion, pentecôtismes, migration, identité, ethnographie.

[Abstract] The purpose of this article is to discuss the methodological and epistemological stakes of a research conducted among a Neo-Pentecostal Christians of the Paris region. The study examines survey conditions research within Neo-Pentecostal Churches mainly attended by Afro-Caribbean migrants and their descendants, highlighting the ambivalence and particularities of the researcher's position. Endeavoring to question the ethnographer's implication regarding religion, the heuristic quality of his or her ethnic, cultural, religious, and eventual social closeness with the investigated environment, the author shows how a method based on self-unveiling and caseby-case work allows to widely rethink the classical notion of "good distance" that is central to the ethnographic relationship.
\end{abstract}

Keywords: fieldwork research, religion, pentecostalisms, migration, identity, ethnography.

\footnotetext{
1 École des hautes études en sciences sociales.
} 


\section{Introduction}

L'univers social que je me propose d'étudier ici est celui des Églises de type néoévangélique (Aubrée, 2003) fréquentées, en majorité, par des migrants africanocaribéens et leurs descendants (Fancello, 2008 ; Maskens, 2009 ; Demart, 2013 ; Mottier, 2010). Celles-ci émergent en France dans les années 1980, face au «besoin d'une poignée de migrants fréquentant à cette époque différentes Églises du protestantisme français, de mener le culte à leur manière : par des chants, les fortes prières, la danse, la musique, les corps» (Mottier, $2010: 154)$.

Marqué par le sceau de la pluralité, ce christianisme venu d'ailleurs s'organise en plusieurs Églises et regroupe des individus cosmopolites, aux profils sociologiques et aux trajectoires sociales hétérogènes, qui évoluent dans des espaces transnationaux. C'est dans cette dynamique concurrentielle qu'il convient de comprendre l'essor desdites Églises dans les recompositions du protestantisme (Boutter, 2011), et la reconfiguration du paysage religieux français (Dejean, 2010). Structurées autour de la « théologie de la prospérité $»^{2}$ (Mariano, 1999; Aubrée, 2000) ces Églises apparaissent à divers degrés comme des espaces de refuge, d'expressions émotionnelles, et surtout de fabrication de nouveaux imaginaires et d'identités nouvelles. Selon une logique de distinction et d'exemplarité, cet ethos inscrit, à divers degrés, le croyant dans un univers polarisé structuré autour d'une logique de lutte spirituelle permanente avec les forces du Mal. Se convertir et adhérer à ces christianismes tendances ${ }^{3}$, c'est

\footnotetext{
${ }^{2}$ Par théologie de la prospérité, il faut entendre une sorte d'alliance plus ou moins tacite entre Dieu et le croyant, qui stipule que plus ce dernier donne son argent à Dieu, voire s'investit au sein de l'Église, plus Dieu le bénira par ses grâces et lui accordera une vie pleine d'abondance (prospérité financière, bonne santé physique et mentale, bonheur, etc.). On pourrait ainsi résumer cette théologie dans ce slogan qu'il est coutume d'entendre dans certaines Églises: «avec Jésus Christ, tu passeras de la pauvreté à la richesse, du manque à l'abondance, du malheur au bonheur, des larmes à la joie, de la maladie à la santé, etc. ». Par ailleurs, puisque nous sommes en présence d'une pluralité d'Églises qui n'ont ni unité liturgique, ni unité théologique, ni unité morale, il conviendrait peut-être de décliner le label, voire la catégorie au pluriel et de parler de théologies de la prospérité, afin de souligner les différents jeux d'appropriation et de réinterprétation à l'œuvre au sien de cette mouvance religieuse.

${ }^{3}$ L'expression «christianismes tendances » renvoie ici au succès que connaissent depuis plusieurs décennies ces Églises issues de la matrice protestante en Afrique subsaharienne notamment, mais aussi en Occident, et en particulier parmi les migrants africains et leurs descendants, qui par leurs pratiques liturgiques (chants, danses, musiques, pratiques corporelles et vestimentaires spécifiques, valorisation des théologies de la prospérité, de l'efficacité de l'action divine, donc des manifestations du Saint Esprit au travers des séances de guérison, de délivrance, de nouveaux rapports à la société d'accueil, à l'autre et à soimême, etc.) viennent directement concurrencer les Églises dites classiques ou historiques (catholique, orthodoxe et protestante classique).
} 
s'approprier dans une logique dichotomique, de nouveaux registres individuels et collectifs, discursifs et pratiques, oscillant entre un avant et un après, entre subjectivité et contraintes communautaires (Pons, 2013). L'acquisition de toutes ces dispositions chrétiennes doit être pensée en termes de processus, qui passe par un véritable travail sur soi quotidien. C'est progressivement que le croyant aidé par l'Esprit Saint et l'ensemble des membres de l'Église se sépare de ce « vieil homme » et intègre de nouvelles normes et valeurs, de nouvelles techniques langagières, corporelles, mais aussi sexuelles.

La question de la « sexualité est un enjeu crucial. [...] La conversion pentecôtiste - et sa conséquence en termes d'apprentissage de technique narrative particulière par les fidèles - n'est pas sans incidence sur leur biographie sexuelle qui devient alors l'objet d'un travail de réécriture » (Maskens, 2011 : 3). Comment expliquer la rareté de données sur la sexualité de ces sujets croyants en France? Dans ces espaces religieux, l'accès aux informations de qualité lorsqu'on n'est pas soi-même converti.e n'est pas chose aisée. L'injonction à la conversion vient souvent ponctuer les différents échanges, ce qui peut devenir très vite embarrassant (Fancello, 2008).

J'ai suivi entre 2013 et 2016 plusieurs de ces Églises. C'est en m'intéressant à l'articulation des questions de migration, de genre, de classes sociales et de construction de croyances que la question de la sexualité a bousculé mon enquête. La problématique de la sexualité, c'est-à-dire sa place et sa fonction, les conditions dans lesquelles un croyant peut ou ne peut pas en jouir et selon quelles modalités, est omniprésente dans ces espaces religieux. Penser le sexuel dans ces Églises et les conditions d'accès à un tel registre, telle est donc l'ambition de cet article. Enquêter sur la sexualité de ces croyants conduit à un constat ambigu : comment parler de sexualité dans un univers où le sexe est généralement un thème tabou, et où l'injonction à l'abstinence avant le mariage et aux rapports sexuels sains dans les couples mariés sont de mises? Comment une socio-anthropologue ancienne pentecôtiste et néopentecôtiste est-elle parvenue à négocier sa présence, et même à obtenir une certaine liberté de travail et des données intimes sur la sexualité/non-sexualité de l'Autre converti, dans un tel milieu prosélyte, où les pressions pour la conduire à se reconvertir et où les demandes en mariage furent nombreuses et sans relâche ? Comment parvenir à obtenir des confidences sexuelles lorsqu'on est une femme dans un monde régi par des rapports genrés ? Comment décrire et analyser sous un regard nouveau des pratiques qui étaient autrefois les siennes ? Comment passer du statut d'insider à celui d'outsider, d'observatrice éclairée ? Dans quelles mesures mon habitus, ma socialisation et ma couleur de peau peuvent être éclairants pour comprendre, dans une perspective réflexive, les conditions d'enquête menée dans le monde des pentecôtismes ?

Mon objectif est de décrypter a posteriori, les avantages et les inconvénients d'une ethnographie menée en terrain "miné », sur une culture religieuse d'une "étrange familiarité »(Ouattara, 2004) que j’avais abandonnée mais dont je maitrisais encore les codes, l'art de faire surtout. 


\section{La relation d'enquête : un contrat à cœurs ouverts}

\subsection{L'épreuve de la confrontation à soi}

La tradition ethnographique " a doté d'une légitimité durable, dès le début du $\mathrm{XX}^{\mathrm{e}}$ siècle, à travers notamment les œuvres de Bronislaw Malinowski, d'Edward EvansPritchard ou de Magaret Mead, les observations de terrain intégrées dans une totalité culturelle » (Dodier, Baszanger, 1997: 37). Cette tradition du terrain ethnographique comme voyage physique et symbolique se retrouve aujourd'hui dans l'idée que pour comprendre un objet il faut y être extérieur. L'extériorité culturelle vis-à-vis de l'objet d'enquête serait un gage de scientificité dans une tradition de recherche française craignant toute forme de collusion chez le chercheur, entre son « appartenance religieuse» et sa «raison scientifique». Pourtant, parmi les fondateurs de la sociologie des religions, beaucoup ont d'abord fréquenté l'Église avant d'en devenir des observateurs éclairés. Pour Sandra Fancello (2008), « les formes d'expression et les implications quotidiennes d'une religiosité “du tout de la vie” amènent l'ethnologue à travailler sans affinité avec le milieu étudié, voire avec d'autant plus de distance, et parfois même de "crispations", que les pressions pour le conduire à se convertir sont nombreuses et sans relâche» (p. 65). Enquêter sur des acteurs religieux dont l'idéologie prosélyte n'est plus à démontrer peut s'avérer source de difficultés (Boumaza, Campana, 2007). Après en avoir fait les frais, Fancello (2008) y voit même dans ce qu'elle qualifie d'entreprise éprouvante, l'épreuve de l'altérité culturelle par excellence.

Dans mon cas, il s'agit moins d'effectuer un voyage vers l'inconnu, en m'immergeant dans un contexte socio-culturel et ethnolinguistique identifié comme radicalement différent du mien, que de revenir, après des années d'absence, dans un microcosme dans lequel une partie de ma socialisation s'est effectuée. J'ai travaillé parmi les «miens», puisqu'il ne s'agit pas ici « d'examiner les pratiques de cet Autre venu d'ailleurs et devenu voisin et dont les mœurs religieuses dérangent ou inquiètent les autorités » (Maskens, 2013: 13) mais d'étudier la religiosité d’individus avec qui je partage des origines ethnoculturelle et religieuse commune.

Étant d'origine gabonaise, convertie au christianisme, ancienne pentecôtiste puis néo-pentecôtiste très engagée, je partageais plusieurs caractéristiques avec mes interlocuteurs, la même foi, la "condition d'immigrée", voire la "condition noire » (Ndiaye, 2008). M'étant convertie au pentecôtisme jeune ${ }^{4}$, à l'âge de 11 ans, j’ai réguliè-

${ }^{4}$ Je tiens à souligner qu'après plusieurs années d'adhésion au système symbolique du Bwete Missoko, ma mère, deux de mes frères (aujourd'hui pasteurs) et moi-même nous sommes convertis au pentecôtisme. Mon père étant maitre-initiateur de Bwete Missoko et ma mère diaconesse dans une Église pentecôtiste, j'ai été socialisée au sein d'une famille dans laquelle la norme quotidienne ne pouvait s'appréhender en dehors de pratiques cultuelles. À l'âge adulte, après avoir intégré une Église de type néo-pentecôtiste, j'ai suivi une 
rement fréquenté ces espaces religieux dans lesquels j'y ai ensuite occupé des responsabilités à forte charge symbolique et exercé des " dons " sous les statuts de "Prophétesse des Nations » et "d'Intercesseuse ». Bien que cette tradition de voyage physique et/ou symbolique et d'immersion dans une culture religieuse complètement étrangère persiste encore aujourd'hui, que se passe-t-il lorsque l'ethnographe partage les codes, règles et valeurs de ses enquêtés ? Le lien de proximité intense du chercheur.e avec la culture religieuse qu'il étudie, voire avec les représentations et pratiques des enquêtés n'est presque jamais restitué, questionné et analysé.

\subsection{Entre insider et outsider: dévoilement de soi, reconversion temporelle ${ }^{6}$ et gestion stratégique d'un double statut}

En négociant mon entrée sur le terrain, j’ai souhaité adopter une démarche transparente pour parer aux éventuels malentendus et problème d'éthique, exposant les raisons de ma présence et mon projet scientifique aussi bien aux pasteurs qu'aux fidèles. Malgré tout, ma présence était constamment interprétée en des termes purement religieux; certaines questions revenaient presque systématiquement : " pourquoi as-tu choisi ce sujet? »; «L'as-tu choisi parce que Dieu te l’a demandé? »; « Es-tu toujours dans la foi ou es-tu rétrograde, car ce n'est pas en vain que Dieu t’a envoyé ici ? » ; « Estu pour nous ou contre nous?». Face à ces questions, l'orientation de mes réponses était structurée autour du désir de connaître les raisons pour lesquelles j'étais soumise à un tel interrogatoire, et celui de savoir si le fait d'être convertie pouvait avoir une incidence sur l'acceptation ou le refus dudit projet. Contrairement à mes attentes, mes questions semblaient agacer mes interlocuteurs, qui se refusaient de croire que je puisse être confessionnellement neutre. Très vite, j’ai compris que, malgré la répétition de mes déclarations, pour mes interlocuteurs, en tant que jeune femme catégori-

formation au pastorat féminin. Cette proximité non refoulée avec le terrain a teinté, durant toute la durée de l'enquête, les rapports enquêtés-enquêtrice d'un non-formalisme que les mois, voire les années de relations assidues de tout ethnographe extérieur à cet univers ne parviennent pas toujours à dépasser.

${ }^{5}$ Il s'agit des charismes au sens de charisma.

${ }^{6}$ Par reconversion temporelle, il faut entendre le fait que pendant toute la durée de mon enquête de terrain, c'est-à-dire trois ans, j'ai décidé de me ré-imprégner, par intermittence, de l'ethos néo-pentecôtiste, en respectant et en appliquant, tout comme mes enquêtés, et non sans "tiraillements ", les codes, normes, valeurs et manières de faire que cet univers de sens sous-tend. C'est une politique de terrain qui, en supposant de manière quasiment simultanée implication et réflexivité, est faite de restrictions et de ruptures, de (re)implication et de (re)engagement, de proximité et de distance. En prétendant rendre compte d'un ancien "réel pour soi » devenu «réel des autres», cette méthode, qui dans une perspective compréhensive, dialogiste et pragmatique fait de la réflexivité permanente un véritable outil de travail, a consisté à «faire comme ces autres", en me re-insérant pleinement dans la vie religieuse et hors religieuse de mes enquêtés. 
sée comme noire, j’avais forcément fait l'expérience de la conversion au cours de ma vie. Et, si mon projet était orienté sur leurs Églises, ce n'était pas en vain. L’intention affichée de certains de m'inclure dans une "communauté imaginaire» (Anderson, 1991), voire dans un panafricanisme chrétien, en particulier néo-pentecôtiste, ne faisait aucun doute. Voici, à titre d'exemple, les propos du pasteur Katoumbi du Centre d'Accueil et de Vię, une Église néo-pentecôtiste majoritairement fréquentée par des individus issus des classes les plus modestes :

Sœur Pamela, ta présence dans notre Église n'est pas le fruit du hasard. À coup sûr, c'est l'oeuvre de Dieu. C'est Dieu qui a guidé tes choix, car il a des plans pour toi et souhaite t'utiliser pour que tu puisses les accomplir?. Ne te laisse pas détourner par la science et son esprit de rébellion! II faut que tu utilises tes connaissances pour l'oeuvre de Dieu. II faut que tu utilises tes connaissances pour aider le peuple de Dieu.

L'adoption d'une casquette de socio-anthropologue "neutre» que je m'efforçais de maintenir à chacun de mes passages sur le terrain était inadaptée, infructueuse et suscitait la méfiance de mes interlocuteurs?. Face à leurs réticences, il fallait trouver une autre voie d'entrée pour m'assurer l'accès aux données dans ce milieu qui n'avait cessé d'être si peu accueillant à ma curiosité; certains pasteurs étaient même allés jusqu'à me dissuader de poursuivre ma recherche. Les pressions pour me pousser à me reconvertir étaient nombreuses et sans relâche. La reconversion temporelle - au risque de perdre mon autonomie de chercheure - semblait s'imposer, ma recherche allait connaître alors un tournant épistémologique (Maskens, 2013).

Tachant de contourner ce problème immanent à ce statut académique d'observatrice, j'ai révélé à chacun des dirigeants et fidèles des Églises enquêtées que, d'une part, je m'étais convertie et avais "grandi dans l'Église »; d'autre part, j'avais pris mes distances avec la religion et étais devenue ce qu'on appelle dans le jargon des pentecôtismes une «chrétienne rétrograde $»^{10}$. Cette entrée par le dévoilement de soi a eu le mérite de libérer la parole sur des sujets variés, de me faciliter l'accès à des espaces et rencontres parfois réservés aux membres, et d'être invitée en tant que « sœur en Christ » aux domiciles de plusieurs d'entre eux. Cela m’a permis non seulement d'avoir accès à l'intimité de plusieurs membres, mais aussi de poursuivre avec préci-

\footnotetext{
${ }^{7}$ Pour respecter l'anonymat des différents interlocuteurs, les noms d'Églises ainsi que ceux des enquêtés ont été modifiés et apparaîtront sous des noms d'emprunts.

${ }^{8}$ Entretien réalisé en mars 2014.

${ }^{9}$ La parution de plusieurs rapports parlementaires de la Mission interministérielle de vigilance et de lutte contre les dérives sectaires (MIVILUDES) a contribué à stigmatiser ces Églises et à développer la méfiance de ces croyants.

${ }^{10}$ Les Églises dans lesquelles j'ai effectué mon terrain ne sont pas les mêmes que celles dans lesquelles ma socialisation religieuse s'est faite.
} 
sion mon enquête sans éveiller les soupçons d'infiltration ${ }^{11}$ de mes interlocuteurs. Comme si le fait d'avoir accepté de réinvestir cette ancienne facette de mon identité leur avait permis de ne plus voir en la socio-anthropologue que je m'efforçais d'incarner une menace potentielle.

C'est donc en jouant la carte du dévoilement de soi, de la mise à nu, que j'ai pu gagner la confiance aussi bien des dirigeants que des simples fidèles et m'inscrire dans leur familiarité non sous le seul statut d'ancienne fidèle, mais également sous celui de socio-anthropologue. Ma position n'était ni clandestine ni complètement à découvert, car « bien qu'ayant fait de l'observation à découvert, (mon statut d'observateur était connu de certains et donc susceptible d'être connu de tous), j'étais pour beaucoup l'équivalent d'un[e] observateur[trice] incognito[a], c'est-à-dire un[e] simple participant[e] » (Broqua 2009: 13). Pour les pasteurs, leurs collaborateurs et certains fidèles à qui j'avais déjà exposé mon projet, j'étais tout simplement une « sœur qu'il fallait récupérer».

Les pasteurs et leurs collaborateurs n’ont alors pas hésité à me présenter à chacun des fidèles croisés pour la première fois comme une « sœur sociologue »; présentation que j'ai reprise à mon compte. L'acceptation de cette étiquette a eu le mérite de rassurer mes interlocuteurs sur le sérieux de ma démarche, et surtout sur mes bonnes intentions à leur égard. Ce n'est qu'après plusieurs mois de présence sur le terrain que je me suis rendue compte qu'en présentant mon projet, j’ai été, en fonction de l'Église, assignée à mon insu à trois places distinctes. Pour certains, j'étais une « envoyée de l'ambassade de la République démocratique du Congo ou de la République gabonaise à Paris ", donc une alliée potentielle ; pour d'autres, j'étais tout simplement une « sœur en Christ rétrograde » que Dieu souhaitait restaurer ; et, pour d'autres encore, j'étais une "envoyée des services de l'immigration», donc une ennemie potentielle. C'est l'annonce de mon passé de convertie et la mise en scène de manières de faire propres à l'ethos néo-pentecôtiste ${ }^{12}$ qui a engendré un changement radical d'attitude vis-à-vis de ma recherche, de ma présence et de mes interrogations régulières.

Les retrouvailles entre "frères et sœurs en Christ " étant l'occasion pour chaque croyant de mettre en scène sa foi et son degré de maturité spirituelle, en tant que

\footnotetext{
${ }^{11}$ C'est également au même moment que j'avais décidé de ne plus sortir mon enregistreur et de ne plus prendre les notes pendant les cultes, les réunions ou encore les rencontres extra-religieuses mais seulement une fois rentrée chez moi; ces actes chers à l'ethnographe n'avaient plus rien de naturels pour moi et me paraissaient désormais d'une violence extrême. En ce sens, ce nouveau regard porté à la mise en scène des entretiens sur le terrain rejoint celui de Maité Maskens, spécialiste des pentecôtismes à Bruxelles, pour qui le geste même de le poser l'enregistreur sur la table n'était plus supportable.

12 Mes faits et gestes lors des différentes rencontres cultuelles étaient scrutés; j'étais observée, car les pasteurs voulaient se rassurer que je fusse bien des «leurs »; je devais donc montrer lors des cultes que je maitrisais parfaitement le langage, les codes, normes et valeurs propres à l'ethos (néo)pentecôtiste.
} 
«sœur sociologue », j'ai dû jouer le jeu. Les dimanches je me rendais à l'Église, en m'assurant d'avoir pris ma Bible, mon cahier et mon stylo, et surtout « mon offrande du dimanche »; tous les premiers dimanches du mois je donnais ma dîme. Je participais aux cultes, aux soirées spéciales, aux veillées et réunions de prières, aux mariages, aux baptêmes, aux événements caritatifs, aux enseignements et séminaires de formations. Je me suis totalement re-immergée dans la vie religieuse des Églises que j'ai ethnographiées. Ma position rappelle celle de Claudia Währisch-Oblau (2009), qui souligne: «qu'elle n'assiste pas au culte en qualité de simple visiteuse. Pasteur[e]de l'Église évangélique de Rhénanie, elle est souvent invitée à prendre la parole. Oratrice ou prédicatrice, elle considère cette posture comme un changement de perspectives favorable au développement d'un autre regard sur le phénomène» (p.18, cité par Maskens, 2013 : 17). Ou bien celle de Jane E. Soothill (2007: 6, citée par Fancello, 2008 : 13) qui se définit comme exevangelical Christian et souligne: «I prayed with them, sang and danced with them, and even sang sometimes in their choir [...] paid my monthly dues ». Ma démarche pose la question des liens entre recherche scientifique et implication au sein des groupes enquêtés. Sa spécificité réside dans ce mouvement de va-et-vient entre le statut de « sœur en Christ » et celui de socio-anthropologue, dans cette tension entre ré-imprégnation quotidienne d'un ethos croyant et autoréflexivité permanente, qui «se présente comme l'association d'une proximité de l'action et d'une distance dans l'analyse » (Fassin, 1999: 54). Malgré ce rôle de «sœur en Christ » que j’ai décidé de réinvestir et cette place de " sœur sociologue » que j'ai acceptée d'occuper ${ }^{13}$, « ma situation a été celle non pas d'un[e] chercheur[e] engagé[e] en tant que tel, mais d'un[e] chercheur[e] procédant à une ethnographie de l'engagement, c'est-à-dire une ethnographie supposant l'engagement au sein du groupe étudié mais l'indépendance et la distance critique, et surtout l'absence de désir d'agir sur le monde social au nom du savoir produit » (Broqua, 2009:10).

Durant tout mon terrain, cette méthode basée sur le dévoilement de soi et la technique du cas par cas a été une expérience à la fois douloureuse et fascinante, d'autant qu'il me fallait penser et réfléchir contre moi-même. Ethnographier ces « semblables » n'est pas rien : il y a dans cet acte en apparence anodin, un arrachement imposé à soimême, qui signale que le soi est publiquement mis à nu, exposé aux regards et aux jugements d'autrui. La relation d'enquête tend ainsi à être un voyage vers le même régi par la loi de l'introspection et de la réflexivité permanente, et surtout soumis aux difficultés de l'objectivité dans un cadre et des conditions qui tendent à imposer la subjectivité. En décidant de me ré-imprégner de cet habitus-croyant, j'étais consciente des

\footnotetext{
${ }^{13}$ Un flou des frontières entre extériorité (socio-anthropologue) et intériorité (« sœur ») qui m'a exigé d'être constamment sur mes gardes, en m'assignant à une position parfois difficilement soutenable. Malgré tout, cette posture facilita au gré d'innombrables malentendus et tours-de main mon intégration en tant que socio-anthropologue, puis la compréhension subjective des manières de faire de mes enquêtés afin de dégager les logiques à l'œuvre.
} 
limites d'un tel parti pris et des questionnements qu'il pourrait générer. Travailler dans un milieu familier parmi des individus avec lesquels on partage une proximité ethnique, culturelle, religieuse, voire sociale, met le chercheur dans une situation extrêmement délicate. Une sorte de double trahison qui ne lui confère, in fine, de part et d'autre, qu'une légitimité partielle. D'un côté, il s'expose au risque d'être suspecté de trahison par les membres de sa «communauté » pour qui un projet de recherche à teneur scientifique peut générer un sentiment d'intrusion suspecte et gênante; et, de l'autre, il risque d'être perçu comme trop proche de ses enquêtés, et soupçonné de manquer de recul nécessaire tant dans l'observation que dans l'analyse des données recueillies, d'autant plus que cette approche du terrain par l'imprégnation est dans une large mesure encore délégitimée dans le champ scientifique. Tout se passe comme si la position de l'ethnographe impliqué a été érigée au rang de position marginale, en particulier parmi les spécialistes du fait religieux en France, et que celui qui brave cet interdit, puis rompt le silence en y mettant des mots, s'expose au risque de se voir taxer d'ethnographe douteux. Sous l'épée de Damoclès, il est donc constamment sommé de justifier son choix et sa méthode de façon cohérente au risque de se voir exclure de la communauté épistémique.

\section{Enquête et jeu de rôles : à la croisée entre genre, classe et couleur de peau}

\subsection{Entre respect et transgression de la charte de la « bonne sexualité »}

La sexualité est un objet de recherche très sensible en ce qu'il touche au domaine de l'intime, à la sphère privée. Dans le monde des pentecôtismes, deux individus ne peuvent jouir légalement de leur sexualité - du moins dans les discours officiels - que dans le cadre du mariage hétérosexuel qui, considéré comme un rite indispensable au bon fonctionnement de l'Église, valide l'idylle amoureuse entre un « frère et une sœur en Christ ». De même, certains comportements et pratiques sexuelles dits "païens » ou diaboliques (Demart, 2013) sont marqués du sceau de l’interdit. Sexualité et procréation étant indissociables, se marier chrétiennement est perçu comme le laisser-passer officiel permettant à chaque fidèle d'assouvir librement, et sous la bénédiction divine, ses élans sexuels.

Face à cette injonction à l'exemplarité sexuelle, à l'abstinence prémaritale, aborder les questions d'ordre sexuel et avoir accès à la parole non officielle, celle des simples fidèles, n'est pas une entreprise aisée. Admettre l'existence de pratiques sexuelles normalement interdites ou non conformes serait reconnaître la fragilité de cette rhétorique de l'interdit, c'est-à-dire "certains brouillages de ce code moral» (Demart, 2013: 789). Il faut souligner avec Sarah Demart (2013) que «l'Église est le lieu d'adhésion à une utopie normative, en construction [...] [qui] peut aussi être un espace privilégié d'hébergement de pratiques, précisément contradictoires avec la norme 
chrétienne » (p. 784). Tout l'enjeu pour ces croyants est donc de respecter au quotidien un écart optimal entre le permis et l'interdit ${ }^{14}$, le normal et l'anormal, le pur et l'impur. Les propos du pasteur Henry d'Impact Centre de Vie sont en ce sens illustratifs. Lors d'un prêche dominical Henry souligne le fait que ses collaborateurs et lui reçoivent très souvent des couples en désaccord sur le plan sexuel, ou encore des femmes qui, mariées avec des "païens », sont obligées par leurs époux d'avoir recours à des pratiques sexuelles interdites par la charte (néo)pentecôtiste. Cela « renvoie aux marges de ce monde religieux, ou pour le dire dans une terminologie foucaldienne, aux espaces 'liminaux' et 'interstitiels' de l'hétérotopie religieuse que constituent les sorties, durables ou éphémères, du monde chrétien » (Demart, 2013 : 785).

C'est en me ré-imprégnant de cet ethos croyant et en adoptant une position d'ethnographe impliquée que j'ai pu avoir accès à la parole des acteurs sur ces expériences cachées. En dépit du discours normatif et de la morale revendiquée, maintenir le cap de l'abstinence ou respecter stricto sensu l'ensemble des interdits ne semble pas être toujours de mise. C'est le cas de Laure qui m'avoue lors d'une de nos conversations entretenir une relation secrète avec un "païen », relation qui se solda par une grossesse. Grégoire, qui à l'époque de notre rencontre était à la recherche d'une « vraie femme », me confia avoir eu deux relations secrètes depuis sa conversion avec deux «païennes ». Ces transgressions soulignent l'existence d'une pluralité des manières d'habiter la norme dans cet univers de sens et la porosité de la frontière entre le «monde païen » et le «monde chrétien », qu'il faut penser en termes de subjectivités d'acteurs et de création de sens pluriels.

Cette logique de contrôle se fait également par le biais de prêches ciblés par catégorie de sexe, censés permettre aux pasteurs de réguler et stabiliser, voire contrôler les pratiques sexuelles des fidèles. Pour donner du relief à cette rhétorique de l'interdit, des réunions des femmes mariées, des hommes mariés, des jeunes femmes, des couples, etc. sont autant d'espaces dédiés à la moralisation du corps. La toilette intime et l'entretien du corps, «le savoir-faire sexuel», la vertu, l'entretien de son domicile, sont souvent les thématiques prisées de ces rencontres. Il existe un discours moralisateur qui vise à encadrer les pratiques sexuelles légitimes, en dictant ce qui doit ou pas être fait lors des jeux sexuels des « corps en action ».

Toutefois, lors des enseignements réservés aux couples mariés, ces derniers sont invités à faire preuve de créativité; malgré cette rhétorique de l'interdit, les croyants sont encouragés à faire jouer leur «sainte imagination »; à demander quotidiennement de l'aide à l'Esprit Saint afin qu'il leur communique une "sainte inspiration » et des « saintes astuces » qui leur permettra de pimenter leur vie de couple. Ainsi, les dirigeants recommandent par exemple aux couples de varier les positions sexuelles ou

14 Par exemple, les couples rencontrant des problèmes d'ordre sexuel sont suivis par d'autres couples responsables et membres du « comité famille ». 
d'érotiser leurs rapports sexuels à l'aide d'huiles essentielles ; parfois, aux femmes, de mettre une petite fleur dans les cheveux, et aux hommes de toujours avoir des beaux caleçons.

\subsection{Ruser pour avoir accès aux confidences sexuelles}

Jouer le jeu de la reconversion dans un univers dans lequel la rhétorique de l'exemplarité vise précisément à se définir en opposition aux autres, impliquait de pouvoir supporter toutes les contraintes qui y étaient associées. Ne pas respecter le code moral et les clauses de la charte de la bonne sexualité était inenvisageable. Pour mes interlocuteurs, j'étais avant tout soit une «fille du pays", soit une "sœur en Christ ", donc une potentielle épouse. J’ai donc été régulièrement questionnée sur ma vie sentimentale et sexuelle ; plusieurs de mes interlocuteurs ne cessaient de me rappeler qu'en tant que jeune femme noire célibataire et surtout « sœur en Christ », je devais me soumettre à l'exigence d'exemplarité sexuelle. Ils rajoutaient généralement que si j'avais des pulsions sexuelles trop fortes, il fallait d'abord que je prie et demande l'aide à l'Esprit Saint pour «tenir ferme », puis, que je me trouve rapidement un fiancé (Millet-Mouity, 2017). M'intéresser à leurs comportements sexuels est très vite devenu l'épreuve de la souffrance. D'autant que les seuls récits et témoignages que j'obtenais étaient ceux de membres qui affirmaient toujours « résister à la tentation" et n'avoir commis aucun écart. Chaque entretien ou discussion informelle déviait d'une part, vers un véritable questionnaire sur ma vie sentimentale et sexuelle; d'autre part, avec les "frères en Christ », vers un jeu de séduction explicite, ces derniers n'hésitant pas à me faire des demandes en mariage. Lorsqu'on est une femme célibataire, enquêter sur les comportements sexuels des converti.es's dans le monde des pentecôtismes peut s'avérer être une source de malentendus variés. Pour échapper à ces interrogatoires successifs et à cette cour récurrente, il a fallu ruser.

Pour dénouer les langues et avoir accès à ces expériences sexuelles nonconventionnelles, j’ai privilégié la technique du cas par cas, en adaptant mon discours sur la sexualité en fonction de mes interlocuteurs et des circonstances. Avec certain.e-s, les «pieux XXL », j'ai valorisé une vie d'abstinence et de strict respect des normes morales; avec d'autres, mon incapacité à résister à la tentation et à contenir mes pulsions sexuelles ${ }^{15}$. J'ai ainsi pu avoir accès à des histoires intimes, à certaines confidences sexuelles sur leurs «moments de faiblesse». Tour à tour, j’ai été soit la parfaite confidente à qui l'on peut confier ces petits secrets, soit la "sœur égarée " qu'il faut à tout prix ramener sur le droit chemin. Valoriser auprès de certains une vie

\footnotetext{
15 Avec ceux qui m'avouaient avoir également connu des «moments de faiblesse » et transgresser la norme à un moment donné de leur parcours chrétien, je pouvais enfin redevenir moi-même et partager ma propre expérience.
} 
non-conforme à l'idéalité des normes, en particulier à l'impératif d'abstinence sexuelle avant le mariage, m’a exposée à de nombreuses critiques. Il était inconcevable qu'une "sœur », fille de diaconesse et petite sœur de pasteur, ayant exercé des responsabilités à forte charge symbolique se perde et adopte la mentalité « dépravée des filles du monde ». Tous mes contacts avec les enquêtés devenaient des mises à l'épreuve, entre diabolisation de mes comportements sexuels et le désir de re-normer, de « re-néo-pentecôtisiser » mon rapport au corps et à la sexualité. Constamment j’ai dû rester sur mes gardes et travailler mon « jeu» pour éviter de me tromper de casquette.

\section{Considérations finales : de la ruse à la saturation}

Si mon agenda de terrain se remplissait, mon moral et ma capacité d'abstinence diminuaient de jour en jour. Chaque passage sur le terrain devenait pour moi une véritable épreuve. Entre ce désir d'être moi-même et celui de faire bonne figure, j'étais prise dans une double tension. D'abord, la peur d'être démasquée par ceux et celles qui refusaient de voir en moi autre chose qu'une "sœur en Christ». Puis, cet étrange sentiment de trahison. Le mélange des deux créait en moi une souffrance morale et psychologique que même le retour à ma réalité hors terrain ne suffisait à apaiser. Cette position en "trait d'union» et surtout tous ces mensonges devenaient insupportables. Pour garantir le bon déroulement de mon enquête, j’ai donc décidé de faire comme tout le monde, en re-expérimentant l'abstinence sexuelle en dehors du cadre conjugal. Un soir, alors que tout semblait aller pour le mieux dans mon couple, j'ai décidé de rompre avec mon compagnon sans explication.

Au bout de quelques mois, je me suis vite rendue à l'évidence: ces mensonges et cette abstinence sexuelle, cette autocensure en somme, exigeaient trop de moi. Il fallait absolument que je me déprenne de ce nouveau «moi » et que je m'extirpe de cet univers qui tendait à me ré-engloutir. Il fallait que je parvienne à m'imposer comme acteur scientifique. Car, il m'arrivait parfois de me tromper de casquette. Dans ces moments d'erreur involontaire, j'étais alors rejetée de mille manières: soit taxée à nouveau de "païenne », de " charnelle » ou encore de « rétrograde »; soit, dans un élan de compassion, invitée à me repentir. Finalement, j’ai décidé de ne plus jouer le jeu et d'annoncer à mes informateurs que j'allais retourner vivre avec mon compagnon. Je venais de réaliser que j'étais arrivée à saturation, et qu'il était temps pour moi de mettre fin à la phase de terrain (Olivier de Sardan, 1995).

Dans le monde des religions, travailler avec affinité pour une ancienne convertie engendre des formes complexes d'implications quotidiennes, et parfois même de compromis et de duperie, tant les pressions pour la conduire à se reconvertir, à redevenir un modèle d'exemplarité sont nombreuses et sans relâche. Soumise à l'épreuve de la ressemblance, elle est souvent sommée de se positionner. Dans mon cas, tout l'enjeu était, en assumant cette proximité avec le milieu enquêté, de parvenir à tra- 
vailler dans l'amitié, avec affinité, tout en gardant l'objectivité nécessaire à toute production scientifique lors de l'analyse de mes données, admettant, dans une approche autoréflexive, que ma position sur le terrain a inévitablement une influence certaine sur les résultats de mon enquête.

\section{Bibliographie}

ANDERSON B. (1991 [1983]), Imagined communities. Reflections on the origin and spread of nationalism, Londres, Verso.

Aubree M. (2000), « La diffusion du pentecôtisme brésilien en France et en Europe : le cas de l'IURD », in C. LERAT, B. RIGAL-CELLARD (dir.), Les mutations transatlantiques des religions, Bordeaux, Presses Universitaires de Bordeaux, p. 149-157.

AubreE M. (2003), «Un néo-pentecôtisme brésilien parmi les populations immigrées en Europe de l'Ouest », Anthropologie et Sociétés, vol. 27, n 1, p. 65-84.

Boumaza M., Campana A. (2007), «Enquêter en milieu difficile. Introduction », Revue française de science politique, vol. 57, $\mathrm{n}^{\circ} 1, \mathrm{p} .5-25$.

BOUTTER B. (2011), « Le protestantisme en France, un terreau d'accueil privilégié pour les migrants ?», in S. FATH, J.-P. Willaime (dir), La nouvelle France protestante, essor et recomposition au XXIe siècle, Genève, Labor et Fides, p. 300-313.

BROQUA C. (2009), "L'ethnographie comme engagement: enquêter en terrain militant », Genèses, vol. 75, n² 2, p. 109-124.

DeJEAn F. (2010), « La précarité spatiale des Églises africaines en Seine-Saint-Denis et sur l'île de Montréal », in S. FANCELLO, A. MARY (dir.), Chrétiens africains en Europe. Prophétismes, pentecôtismes et politiques des nations, Paris, Karthala, p. 301-325.

Demart S. (2013), "Genre et transgression des normes morales et sexuelles dans les Églises de Réveil à Kinshasa et en diaspora ", Cahiers d'études africaines, nº 212 , p. 783-811.

Dodier N., BASZANGer I. (1997), «Totalisation et altérité dans l'enquête ethnographique », Revue française de sociologie, vol. 38, n 1. p. 37-66.

FANCELlo S. (2008), "Travailler sans affinité: l'ethnologue chez les "convertis"», Journal des anthropologues, $\mathrm{n}^{\circ}$ 114-115, p. 65-90.

FASSIN D. (1999), "L'anthropologie entre engagement et distanciation. Essai de sociologie des recherches en sciences sociales sur le sida en Afrique», in C. BECKER et al. (dir.), Vivre et penser le sida en Afrique, Paris, Codesria, Karthala \& IRD, p. 41-66. 
MARIANo R. (1999), Neo-pentecostais : sociologia do novo pentecostalismo no Brasil. São Paulo, Loyola.

MASKENS M. (2009), « Identités sexuelles pentecôtistes : féminités et masculinités dans des assemblées bruxelloises », Autrepart, $\mathrm{n}^{\circ}$ 49, p. 65-81.

MASKENS M. (2011), « Le traitement de la virginité chez les migrants pentecôtistes à Bruxelles », L'Espace Politique [En ligne], 13 | 2011-1, mis en ligne le 06 mai 2011, consulté le 15 octobre 2013. URL : http://espacepolitique.revues.org/1847.

MASKens M. (2013), Cheminer avec Dieu: pentecôtismes et migrations à Bruxelles, Bruxelles, Éditions de l'Université de Bruxelles.

Millet-Mouity P. (2017, à paraître), «De l'entre-soi conjugal. Phénoménologie du mariage chez les néo-pentecôtistes euro-africano-caribéens de la région parisienne», in N. GUILLET (dir), La diversité religieuse en France. Approche pluridisciplinaire, Rouen, Presses Universitaires de Rouen et du Havre.

MotTIER D. (2010), «Réveils prophétiques et entreprenariat charismatique. Creuset congolais et prophétisme ivoirien en France», in S. Fancello, A. MarY (dir.), Chrétiens africains en Europe. Prophétismes, pentecôtismes et politiques des nations, Paris, Karthala, p. 155-178.

NDIAYE P. (2008), La condition noire. Essai sur une minorité française, Paris, Éditions Calmann-Lévy.

OlIVIER DE SARDAN J.-P. (1995), « La politique du terrain. Sur la production des données en anthropologie », Enquête. Anthropologie, Histoire, Sociologie, vol. 1, p. 71-109.

OUATTARA F. (2004), «Une étrange familiarité. Les exigences de l'ethnographie "chez soi" », Cahiers d'études africaines, vol. 2, n 75, p. 635-658.

Pons C. (dir.) (2013), La construction collective d'une relation personnelle à Jésus dans les Églises évangéliques: Europe, Océanie, Maghreb, Paris, CNRS Éditions.

Soothill J. E. (2007), Gender, Social Change and Spiritual Power: Charismatic Christianity in Ghana, Leyde, Brill.

Währisch-Oblau C. (2009), The Missionary Self-Perception of Pentecostal/Charismatic Church Leaders from the Global South in Europe. Bringing Back the Gospel, Leyde, Brill. 